\begin{tabular}{|c|c|c|}
\hline \multirow{2}{*}{$\begin{array}{l}\text { IV Simpósio Paranaense de Modelagem, } \\
\text { Simulaçãoe Controle de Processos } \\
\text { ISSN : 1984-7521 }\end{array}$} & Artigo: & 08 \\
\hline & Páginas & $54-61$ \\
\hline
\end{tabular}

\title{
MODELAGEM E SIMULAÇÃO DE UM REATOR TUBULAR PARA A REAÇÃO DE FORMAÇÃO DO ACETATO DE ETILA
}

\author{
Isabelle Moreira*, Matheus Amplatz Iurk, Rafaella Moreira, Emerson Martim \\ Pontificia Universidade Católica do Paraná, *belle.moreira08@gmail.com
}

\begin{abstract}
Resumo - Estudos que visam otimizar processos industriais são de suma importância para melhorar o mercado, tornando-o mais atrativo, de forma a operar nas condições ótimas que favoreçam a produção. $\mathrm{O}$ acetato de etila, composto químico selecionado para a realização da modelagem, possui amplo campo de utilização, na composição de vernizes, tintas, produtos farmacêuticos, adesivos, esmaltes, dentre outros. O objetivo deste trabalho foi desenvolver a simulação e a modelagem de um reator PFR (plug flow reactor), realizando-se as análises das principais variáveis de processo e condições ótimas de operação. Os balanços de massa e de energia foram realizados, chegando-se a equações diferenciais parciais (EDP) em relação ao tempo e à posição na direção axial. Em estado estacionário resultou em diferenciais ordinárias (EDO). Foram usados parâmetros cinéticos, os quais foram obtidos por meio de experimentos, havendo a verificação de que a reação é elementar de segunda ordem. Também se conseguiu verificar que a constante cinética possui um valor alto, realizando-se a desconsideração da parte inversa. Em relação à modelagem do reator, para o regime estacionário chegaram-se a 24 tubos, com $3 \mathrm{~m}$ de comprimento, cujo raio central é igual a $20 \mathrm{~cm}$ e o raio da região anelar é de $40 \mathrm{~cm}$. Para o estado transiente, foi considerado que o reator é isotérmico, sendo proposta a modelagem a partir de um caso real, obtendo-se 4 equipamentos, cujos comprimentos de cada tubo correspondem a $3 \mathrm{~m}$, e os raios da região central a $60 \mathrm{~cm}$, chegando-se a um total de 24 tubos. Os comportamentos das curvas foram representativos, de forma a proporcionar boa análise das condições. Estudos com o intuito de melhorar etapas produtivas são muito significativos, sendo cruciais para a otimização e valorização de processos.

Keywords: Acetato de etila, PFR, otimização.
\end{abstract}

\section{Introdução}

Ao tratar acerca de otimização de processos, busca-se encontrar a melhor solução (condições ótimas) dentre várias existentes para um problema, partindo-se de critérios previamente instituídos. Para formular a situação, deve-se definir a função que será estudada, e as restrições que deverão ser atendidas.

Para a reação de produção do acetato de etila realizou-se a modelagem em um reator tubular (PFR). Os principais países exportadores de acetato de etila são: China (26\%), Bélgica (20\%), e Reino Unido (14\%), enquanto os principais importadores são: Bélgica (17\%), Itália $(9,1 \%)$ e Japão (8,6\%) [5]. O Brasil apresenta 1,7\% de participação na exportação, o que movimenta aproximadamente $\mathrm{R} \$ 53,4 \mathrm{M}$, e $0,40 \%$ na importação, o que significa aproximadamente R\$ 12,3M [5].

As empresas Rhodia e Cloroetil são majoritariamente responsáveis pela produção de acetato no Brasil, tendo sido, a primeira citada, produtora de $4,1 \%$ de toda a produção em cenário global, no ano de 2009 [4]. A Rhodia, entre 2015 e 2016, foi a maior produtora brasileira de ésteres acéticos, respondendo por $91,8 \%$ da produção do país. A Cloroetil Solventes Acéticos S.A. possui a segunda maior participação, com quase todo o restante da produção nacional [3].

Com esses dados, salienta-se a importância do estudo dessa reação. Em cenário altamente competitivo, tal como foi demonstrado, há uma grande necessidade de melhorar os processos, visando maior produção a um menor custo, e consequentemente, aumentando o lucro. Sendo assim, propôs-se a realização da modelagem em regime estacionário e transiente em um reator tubular, objetivando-se avaliar e simular valores

\section{4 e 15 de março de 2019 Curitiba - Paraná}


para as principais variáveis, de forma a conseguir visualizar os perfis e analisar os comportamentos, obtendo-se resultados acerca das condições ótimas de processo.

\section{Acetato de etila}

O produto escolhido para o estudo, acetato de etila, é um éster, que apresenta alta inflamabilidade, é pouco volátil, incolor, possui ponto de fusão (PF) igual a $-83,75$ ${ }^{\circ} \mathrm{C}$ e ponto de ebulição (PE) de $57,15{ }^{\circ} \mathrm{C}$, sendo líquido nas condições padrões. Possui amplo campo de utilização e aplicação industrial, como na composição de vernizes e tintas, produtos farmacêuticos, adesivos e esmaltes [1] e [6]. O produto pode ser obtido por um processo de esterificação, conforme representado abaixo, a partir de etanol e ácido acético, assim como mostrado na reação.

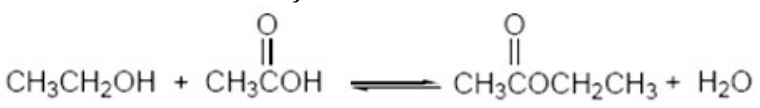

\section{Plug Flow Reactor}

Este reator é formado, geralmente, por um par de tubos concêntricos, escoando a mistura reacional na região central e o fluido de remoção ou fornecimento de calor na porção do anel, para reações não isotérmicas. Constitui-se de um escoamento pistonado ou plug flow (PFR), no qual não ocorrem variações radiais das propriedades, sendo uniforme o perfil radial de velocidade [7].

\section{Metodologia}

\section{Estudo Cinético}

Para realizar o estudo cinético da reação, utilizaram-se os compostos químicos em quantidades iguais a 1,0 L de ácido acético glacial, 1,5 L de álcool etílico absoluto e $120 \mathrm{~mL}$ de ácido sulfúrico PA. Inicialmente, foram colocados no reator o álcool etílico e o ácido sulfúrico. Aqueceu-se a mistura até $60^{\circ} \mathrm{C}$, e em seguida acrescentou-se o ácido acético lentamente, realizando-se o controle de duas variáveis, correspondentes ao tempo e à temperatura, que deveria permanecer na faixa de 70 a $80^{\circ} \mathrm{C}$. Alíquotas da mistura reacional puderam ser coletadas a cada 3 minutos, sendo devidamente depositadas em erlenmeyers, em quantidades de $30 \mathrm{~mL}$. Adicionou-se fenolftaleína em cada frasco, fazendo-se a titulação com solução de hidróxido de sódio $2 \mathrm{M}$.

\section{Balanço de massa global}

A modelagem do reator foi realizada conforme o desenvolvimento descrito a seguir, em que A e B são reagentes limitante (ácido acético) e em excesso (etanol), C é o produto acetato de etila e D a água, também formada na reação.

$$
\begin{gathered}
\text { Sai }=\text { Entra } \pm \text { Reage }- \text { Acúmulo } \\
\frac{d m}{d t}=\rho e q e-\rho s q s
\end{gathered}
$$

Em que: $\rho e$ e $\rho s$ são as massas específicas de entrada e saída, respectivamente, $\left(\mathrm{kg} / \mathrm{m}^{3}\right) ; \mathrm{m}$ - massa $(\mathrm{kg}) ; \mathrm{t}$ - tempo (s) e q - vazão volumétrica $\left(\mathrm{m}^{3} / \mathrm{s}\right)$.

Como a variação de volume é constante ao longo do tempo (reação química em fase líquida), o termo é nulo. As massas específicas são consideradas iguais porque o reator é bem misturado e os fluidos são incompressíveis. 
Balanço de massa do componente A

$$
V \frac{d C a}{d t}=q C a e-q C a-k C a C b V
$$

Em que: V - volume do reator tubular $\left(\mathrm{m}^{3}\right) ; \mathrm{Ca}$ e $\mathrm{Cb}$ - concentrações dos reagentes limitante e em excesso, respectivamente $(\mathrm{mol} / \mathrm{L})$ e $\mathrm{k}$ - constante cinética (L/(mol.s)). A reação em estudo foi considerada como elementar e irreversível.

Sabe-se que o volume pode ser calculado por: $V=\pi R^{2} \Delta Z$, assim como a vazão volumétrica é $q=v \pi R^{2}$, obtendo-se a Equação 04 .

$$
\pi R^{2} \Delta Z \frac{d C a}{d t}=v \pi R^{2} C a e-q C a-k C a C b \pi R^{2} \Delta Z
$$

Em que: $\mathrm{R}$ - raio (m), $Z$ - altura $(\mathrm{m})$ e $v$ - velocidade $(\mathrm{m} / \mathrm{s})$.

Reorganizando a Eq. 04, chega-se à Eq. 05:

$$
\frac{\partial C a}{\partial t}=-v \frac{\partial C a}{\partial z}-k C a C b
$$

Os mesmos passos são realizados para o reagente B (em excesso) e para os produtos $\mathrm{C}$ e D, obtendo-se às Equações 06, 07 e 08.

$$
\begin{aligned}
& \frac{\partial C b}{\partial t}=-v \frac{\partial C b}{\partial z}-k C a C b \\
& \frac{\partial C C}{\partial t}=-v \frac{\partial C C}{\partial z}+k C a C b \\
& \frac{\partial C d}{\partial t}=-v \frac{\partial C d}{\partial z}+k C a C b
\end{aligned}
$$

Balanço de energia

Parte-se da equação geral do balanço de energia, representada por:

$$
\left.E_{T}\right|_{t+\Delta t}-\left.E\right|_{t}=q \rho e_{t e} \Delta t-q \rho e_{t} \Delta t+\dot{Q} \Delta t+\dot{W} \Delta t+\dot{R_{T}} V \Delta t \quad \text { Eq. } 09
$$

Em que: $\left.E\right|_{t^{-}}$conteúdo de energia térmica em determinado instante; $e_{t e}$ e $e_{t^{-}}$ relação do conteúdo energético por unidade de massa na alimentação e na saída, respectivamente, e $R_{T}$ - taxa de liberação de energia térmica $\left(\mathrm{J} /\left(\mathrm{m}^{3} \cdot \mathrm{s}\right)\right)$. Encontra-se a Eq. 10 aplicando-se o limite em que a diferencial de tempo tende a zero, sabendo-se que:

$$
\begin{gathered}
E_{T}=\rho \times e_{t} \times V ; \\
e_{t}=c p \times(T e-T) ; \\
\dot{Q}=U \times A \times(\text { Tanel }-T) ; \\
\dot{R_{T}}=k \times C a \times C b \times\left(-\Delta H_{R}\right) \\
\rho \pi R^{2} \Delta z c p \frac{d T}{d t}=v \rho \pi R^{2} c p\left(\left.T\right|_{z}-\left.T\right|_{z+\Delta z}\right)+U 2 \pi R \times \Delta z(\text { Tanel }-T)+ \\
k C a C b\left(-\Delta H_{R}\right) \pi R^{2} \Delta z \\
\text { Eq. } 10
\end{gathered}
$$

Em que: Te e $\mathrm{T}$ - temperaturas de entrada e saída, respectivamente (K); U coeficiente de troca térmica $\left(\mathrm{W} /\left(\mathrm{m}^{2} . \mathrm{K}\right)\right)$ e Tanel - temperatura do fluido no anel $(\mathrm{K})$.

O termo do trabalho foi desprezado por ser muito inferior aos demais. A energia química é transformada em energia térmica proporcionalmente à velocidade de 
reação, correspondendo, este coeficiente de proporcionalidade, ao calor de reação $\left(-\Delta H_{R}\right)$, que recebe o sinal negativo por tratar-se de uma reação exotérmica.

$$
\rho c p \frac{\partial T}{\partial t}=-v \rho c p \frac{\partial T}{\partial z}+\frac{2 U}{R}(T \text { anel }-T)+k \operatorname{CaCb}\left(-\Delta H_{R}\right) \quad \text { Eq. } 11
$$

$\mathrm{Na}$ região anelar, porção em que ocorre a retirada de calor do sistema tem-se a Eq. 12, na qual os índices representados por A, que acompanham as variáveis na Eq. 12, indicam os parâmetros do fluido de resfriamento, que no caso é a água.

$$
\frac{\partial T_{A}}{\partial t}=\frac{-w}{\rho_{A} \pi R_{\text {anel }}^{2}} \frac{\partial T_{A}}{\partial z}+\frac{2 U\left(T-T_{A}\right)}{\rho_{A} R_{\text {anel }} C p_{A}}
$$

Em que $R_{\text {anel }}$ é o raio da porção anelar, em $\mathrm{m}$.

\section{Modelagem em Estado Estacionário}

Desconsideram-se os termos que variam em relação ao tempo nas equações 58, 11 e 12, obtendo-se um conjunto de EDOs.

\section{Modelagem em Estado Transiente}

Para a resolução das equações diferenciais parciais, utilizou-se o método das diferenças finitas. A equação para o termo A pode ser escrita da seguinte forma, após reescrever a concentração de $\mathrm{B}$ em função do reagente limitante e ao introduzir os termos adimensionais:

$$
\left(\mathrm{A}^{*}=\mathrm{Ca} / \mathrm{Cai} ; \mathrm{B}^{*}=\mathrm{Cb} / \mathrm{Cai} ; \mathrm{C}^{*}=\mathrm{Cc} / \mathrm{Cai} ; \mathrm{D}^{*}=\mathrm{Cd} / \mathrm{Cai} ; \Theta=\mathrm{t} / \tau ; \xi=\mathrm{z} / \mathrm{L} \text { e } \tau=\mathrm{L} / \vartheta\right)
$$

$$
\frac{\partial A^{*}}{\partial \theta}=-\frac{\partial A^{*}}{\partial \xi}-k A^{*} \tau\left(C b i-C a i+A^{*} C a i\right)
$$

Faz-se, em seguida, a discretização, no tempo e no espaço. Utilizando as definições da derivada (Eq. 14 e 15), chegando-se à Eq. 16.

$$
\begin{aligned}
& \frac{\partial A^{*}}{\partial \theta}=\frac{A^{*}{ }_{i, j+1}-A^{*}{ }_{i, j}}{\Delta \theta} \\
& \frac{\partial A^{*}}{\partial \xi}=\frac{A^{*}{ }_{i, j}-A^{*}{ }_{i-1, j}}{\Delta \xi}
\end{aligned}
$$

Em que o índice i corresponde à posição e j ao tempo.

$$
A^{*}{ }_{i, j+1}=\frac{\Delta \theta}{\Delta \xi} A^{*}{ }_{i-1, j}+A^{*}{ }_{i, j}\left[1-\Delta \theta\left(\frac{1}{\Delta \xi}+k \tau\left(C b i-C a i+A^{*}{ }_{i, j} \text { Cai }\right)\right)\right] \quad \text { Eq. } 16
$$

O mesmo processo foi realizado para $\mathrm{B}, \mathrm{C}$, e $\mathrm{D}$, chegando-se às Eq. 17 - 19.

$$
\begin{aligned}
& B^{*}{ }_{i, j+1}=\frac{\Delta \theta}{\Delta \xi} B^{*}{ }_{i-1, j}+B^{*}{ }_{i, j}\left(1-\frac{\Delta \theta}{\Delta \xi}\right)-k \tau A^{*}{ }_{i, j} \Delta \theta\left(C b i-C a i+A^{*}{ }_{i, j} \text { Cai }\right) \\
& C^{*}{ }_{i, j+1}=\frac{\Delta \theta}{\Delta \xi} C^{*}{ }_{i-1, j}+C^{*}{ }_{i, j}\left(1-\frac{\Delta \theta}{\Delta \xi}\right)+k \tau A^{*}{ }_{i, j} \Delta \theta\left(C b i-C a i+A^{*}{ }_{i, j} \text { Cai }\right)
\end{aligned}
$$




\section{Resultados e discussões}

\section{Estudo cinético}

A modelagem foi realizada de acordo com considerações como: volume constante ao longo do tempo, fluidos bem misturados e incompressíveis, reação irreversível e elementar. Isso porque, de acordo com a equação de relação entre o número de Gibbs e a constante de equilíbrio, encontrou-se valor de $\mathrm{K}$ de 8,9402 à temperatura ambiente, indicando uma constante de velocidade direta muito superior à inversa. Por isso foi desconsiderada a parte inversa da reação. Também se destaca que o estudo cinético, realizado em batelada, foi fundamental para a verificação da ordem e obtenção dos dados, entretanto, a modelagem é feita para um reator contínuo.

Analisaram-se os dados apanhados a partir do experimento do estudo cinético, sendo possível avaliar a ordem da reação. Utilizou-se o método diferencial por meio das equações padrões (Eq. 20 - 22) [2], chegando-se à uma reação de segunda ordem, em que o coeficiente de determinação $\left(\mathrm{R}^{2}\right)$ resultou em 0,988 .

$$
\begin{aligned}
\text { Ordem 0: } C_{A} & =-K \cdot t+C_{A 0} \\
\text { Ordem 1: } \ln \left(C_{A}\right) & =-K \cdot t+\ln \left(C_{A 0}\right) \\
\text { Ordem 2: } \frac{1}{C_{A}} & =-K \cdot t+\frac{1}{C_{A 0}}
\end{aligned}
$$

A partir da relação da equação obtida pela curva resultante, chegou-se à constante de velocidade a $70^{\circ} \mathrm{C}$, cujo valor é de $0,216 \mathrm{~L} /(\mathrm{mol}$.min). A entalpia também foi calculada, resultando em $-5880 \mathrm{~J} / \mathrm{mol}$, o que indica que a reação é exotérmica, enquanto que a energia livre de Gibbs, igual a $-5430 \mathrm{~J} / \mathrm{mol}$, mostra a espontaneidade. Com esses valores, obteve-se a constante de equilíbrio nas condições padrões, igual a 8,940 , enquanto que na condição operacional, é de 6,550.

Para determinar a energia de ativação, calculou-se a constante de velocidade. Foi considerado o estudo de Kistiakawshy, chegando-se a 0,001 L/(mol.min). A energia de ativação foi encontrada, sendo igual a 101605,52 J/mol, a partir da relação do valor da constante de velocidade obtido experimentalmente. A constante de Arrhenius (ko) é de $1,0455 \times 10^{10} \mathrm{~m}^{3} /(\mathrm{mol} . \mathrm{s})$. O gráfico da ordem foi obtido no Excel.

\section{Estado estacionário}

A partir das equações obtidas com a desconsideração da derivada parcial em relação ao tempo, foram gerados os gráficos em função da posição. Considerou-se a velocidade constante para os cálculos, visto que tanto a vazão volumétrica quanto a área de seção transversal serão mantidas em todo o trajeto do fluido reacional no equipamento. As simulações em regime estacionário, assim como os gráfícos bidimensionais contidos no trabalho, foram feitos em Python.

A partir dos valores de concentração inicial dos reagentes de $1000 \mathrm{~mol} / \mathrm{L}$ para A e $1500 \mathrm{~mol} / \mathrm{L}$ para $\mathrm{B}$, e da constante de velocidade (k), determinaram-se as curvas que regem o comportamento das concentrações em relação à direção axial (Figura 1). E com os dados de concentração do limitante $(\mathrm{Ca})$, construiu-se a curva que demonstra o comportamento da conversão ao longo da direção axial (Figura 2). 
Figura 1-Curva de concentração x posição no reator em regime estacionário

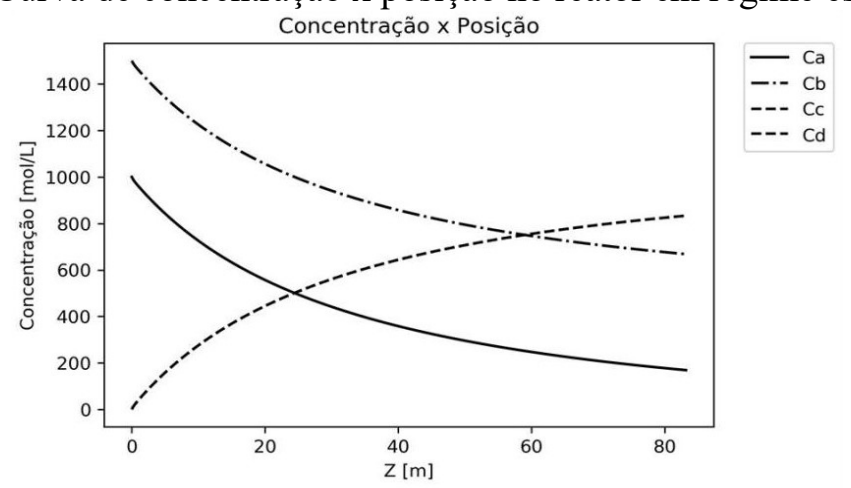

Figura 2 - Conversão do reagente limitante x posição no reator em regime estacionário Conversão x Posição

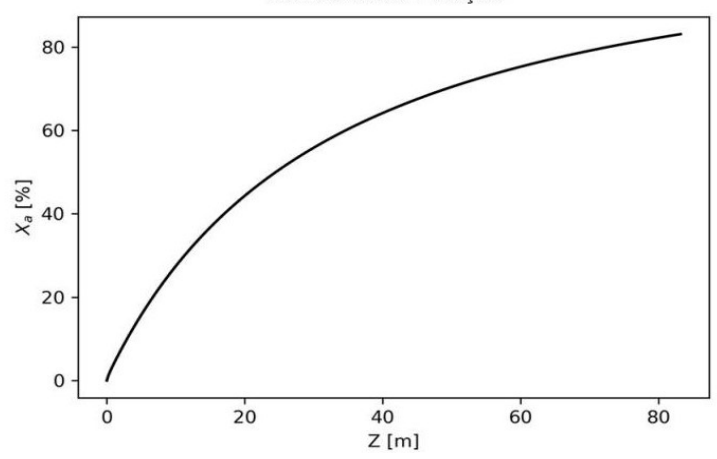

Assim como esperado, a concentração dos reagentes limitante (A) e em excesso (B) decaíram, apresentando curva mais acentuada até próximo a 72 metros. Após esse ponto, a concentração dos produtos não é aumentada de forma tão representativa na medida em que o fluido percorre o reator. A uma posição na direção axial igual a 72 metros, a conversão corresponde a $79,1 \%$, e o erro relativo, pelo método de Runge-Kutta de $4^{\mathrm{a}}$ ordem, é de apenas $0,32 \%$, chegando-se a uma boa aproximação. Em regimes estacionários, como não são considerados os termos de acúmulo, tem-se que as variáveis dependem exclusivamente da posição. Caso os tubos possuam os tamanhos convencionalmente encontrados, como por exemplo, 3 metros de comprimento, o reator industrial seria constituído por um total de 24 tubos, com raio central de $20 \mathrm{~cm}$ e anel cujo raio é de $40 \mathrm{~cm}$.

A partir do balanço de energia, chegaram-se às equações de temperatura do fluido reacional e do fluido de resfriamento em relação à posição, obtendo-se a Figura 3.

Figura 3 - Temperaturas no interior do reator e no anel x posição em regime estacionário

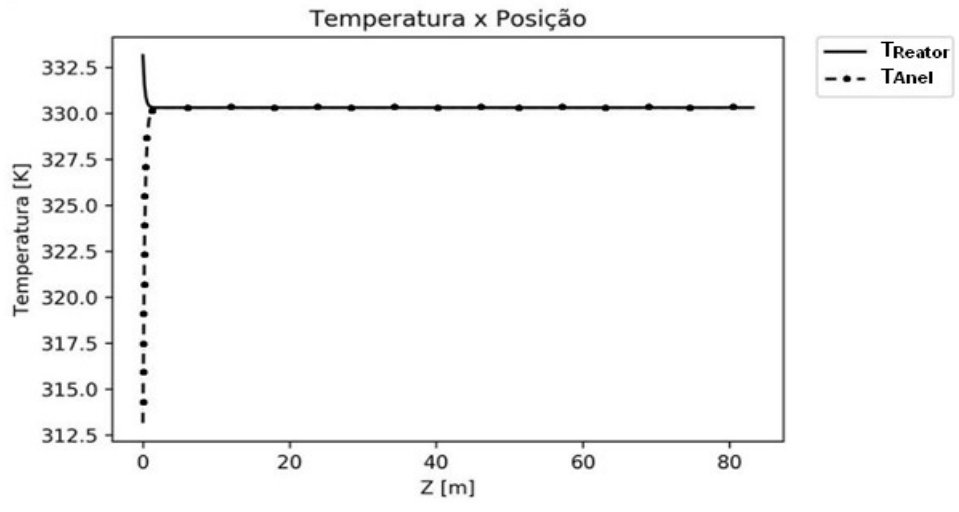

Verificou-se que as temperaturas rapidamente entram em equilíbrio. Esse comportamento não seria tão imediato com a mudança de alguns parâmetros, como o 
coeficiente global de troca térmica (U). Caso este valor fosse inferior, as curvas encontrar-se-iam em uma posição maior. Além disso, alterações na velocidade, variação de temperatura e raio do reator também seriam fatores que influenciariam na posição de convergência dos pontos.

Nessas condições, tanto a temperatura do fluido reacional como a temperatura do fluido de refrigeração é igual a 330,03 K. Isso ocorre também conforme o esperado, visto que a temperatura no interior do tubo central inicia mais alta, na temperatura operacional, e é reduzida à medida que há a transferência de calor com o fluido que percorre o anel. Este, por sua vez, entra a uma temperatura mais baixa, tendo seu valor aumentado, até que ambos encontram o equilíbrio. A vazão de água (4 $\mathrm{kg} / \mathrm{s})$ foi estipulada, de forma a promover boa remoção de calor, não deixando que a temperatura do reator possa aumentar muito e, consequentemente, prejudicar a formação de produtos, já que se trata se uma reação exotérmica.

\section{Estado transiente}

A partir das equações discretizadas, determinadas pelo método de diferenças finitas, obtiveram-se os gráficos correspondentes às variáveis adimensionais.

Os comportamentos podem ser visualizados nas Figuras $5-7$, de acordo com cada uma das cinco divisões realizadas no reator (Figura 4).

Figura 4 - Divisões do reator PFR

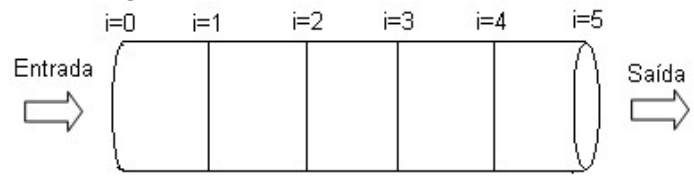

Figuras 5 e 6-Comportamento em regime transiente para os reagentes A e B
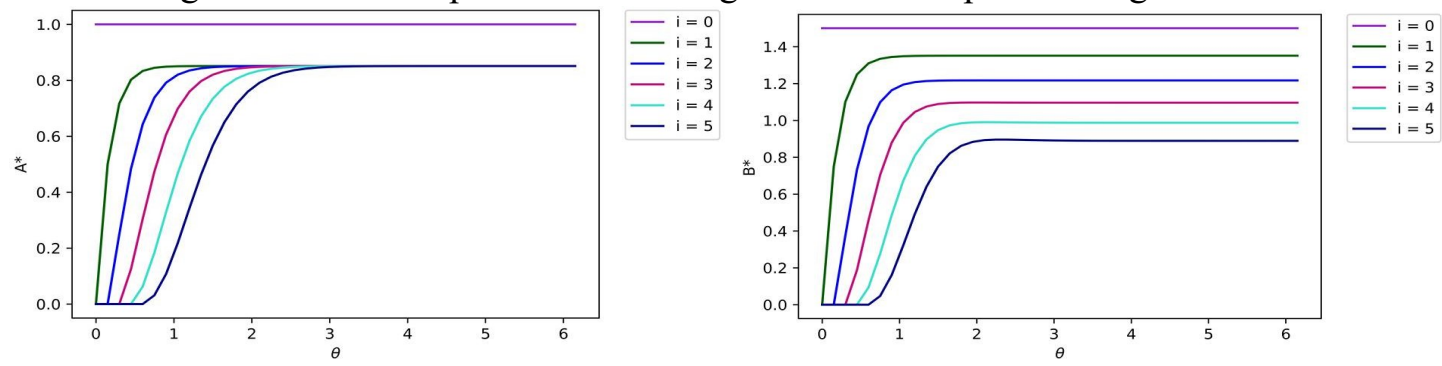

Figura 7 - Comportamento em regime transiente para os produtos C e D

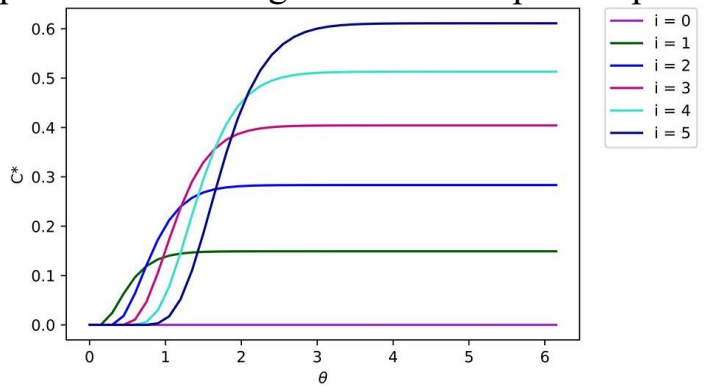

Os comportamentos dos produtos $\mathrm{C}$ e $\mathrm{D}$ foram os mesmos, o que já era previsto, devido à estequiometria da reação, obtendo-se concentração de $0,611 \mathrm{~mol} / \mathrm{L}$. Todas as curvas tornaram-se constantes após $\Theta$ assumir valor de 3,15, para um tempo de residência no reator de 3 minutos. 
As concentrações dos reagentes possuem a primeira curva $(i=0)$ constante, $o$ que ocorre devido à condição de contorno, que implica que a concentração é máxima na entrada do reator, para qualquer $\Theta$. Para os produtos, por outro lado, essa quantidade é nula, pois nada foi produzido nesse momento. Já na condição inicial, para qualquer posição do reator, tanto a quantidade de reagentes quanto de produtos é nula.

Para avaliar um caso real, utilizaram-se os dados da Rhodia, que produzia, em 2008, 131000 ton/dia de acetato de etila (MELLO JÚNIOR, 2010). Utilizando-se um raio de $0,60 \mathrm{~m}$, chega-se a uma velocidade de $1,49 \mathrm{~m} / \mathrm{s}$. O comprimento do reator, a um tempo de residência de 193 segundos, é de aproximadamente $288 \mathrm{~m}$. Como o comprimento é grande, pode-se fazer um arranjo de reatores tubulares. Empregando-se 4 reatores na indústria, com comprimento dos tubos de $3 \mathrm{~m}$, tem-se um total de 24 tubos em cada equipamento. Esta avaliação deve ser feita conforme necessidade de produção.

\section{Conclusões}

Em relação ao regime estacionário, verificou-se a partir dos gráficos apresentados que as curvas começaram a comportar-se de forma menos acentuada a partir de 72 metros na direção axial. Com isso, foi estipulado que poderia haver um total de 24 tubos, com 3 metros de comprimento cada um, cujo raio central é de $20 \mathrm{~cm}$, e a região anelar compreende $40 \mathrm{~cm}$. Nesse ponto, as temperaturas dos fluidos reacionais e de resfriamento são iguais, possuindo valor de 330,03 K. Com a simulação em regime transiente, propôs-se a aplicação da modelagem em um caso real, utilizando-se a capacidade produtiva da empresa Rhodia no ano de 2008. Com isso, obtiveram-se 4 equipamentos, cujos comprimentos de cada tubo correspondem a $3 \mathrm{~m}$, e os raios da região central a $60 \mathrm{~cm}$, chegando-se a um total de 24 tubos por reator.

\section{Referências}

[1] EMBRAPA. Acetato de etila. Disponível em: $<$ http://www.agencia.cnptia.embrapa.br/gestor/agroenergia/arvore/CONT000 fbl23vn102wx5eo0sawqe333t7wt4.html>. Acesso em: $02 \mathrm{dez} 2017$.

[2] LEVENSPIEL, O. Engenharia das reações químicas. São Paulo: Editora Edgard Blücher, $3^{\mathrm{a}}$ ed. Ed. da Universidade de São Paulo, v. 2, 2000.

[3] MDIC. Circular $\mathbf{n}^{\mathbf{0}}$ 58, de 15 de setembro de 2016. Disponível em: $<$ http://www.mdic.gov.br/images/REPOSITORIO/secex/gab/circulares_secex_20 16/circ_secex_058_2016.pdf>. Acesso em: 03 dez. 2017.

[4] MELLO JÚNIOR, D. C. Produção de acetato de etila em biorrefinaria, uma análise de viabilidade. $2010.59 \mathrm{f}$. Dissertação (mestrado) - Escola de Economia de São Paulo, Fundação Getúlio Vargas, São Paulo, 2010.

[5] OEC. Ethyl Acetate. Disponível em: $<$ https://atlas.media.mit.edu/en/profile/hs92/291531/>. Acesso em: 03 dez. 2017.

[6] PERRY, R. H.; GREEN, D. W. Perry's Chemical Engineers' Handbook.6 ${ }^{\mathrm{a}}$ ed., Nova Iorque, McGraw-Hill Book Co., 1984.

[7] PINTO, J. C.; LAGE, P. L. C. Métodos Numéricos em Problemas de Engenharia Química. Rio de Janeiro: E-papers, 2001. 